\title{
A SHORT PROOF OF THE DAWKINS-HALPERIN THEOREM
}

\author{
DAVID HANDELMAN
}

\begin{abstract}
A brief proof is presented, of the Dawkins-Halperin Theorem, that if $D$ is a finite dimensional division algebra with centre $F$, then the direct limits of appropriately-sized matrix rings over $D$ and $F$ are isomorphic; the isomorphism can be given in a form suitable for comparing cohomology groups of $D$ and $F$.
\end{abstract}

For a ring $R$, we denote the ring of $t$ by $t$ matrices with entries from $R$, by $M_{t} R$. There is an obvious map from $R$ to $M_{t} R$ :

$$
\Delta(t): R \rightarrow M_{t} R, \quad r \mapsto\left[\begin{array}{lllll}
r & & & & \\
& r & & & \\
& & \ddots & & \\
& & & r & \\
& & & & r
\end{array}\right] .
$$

Let $D$ denote a division ring of dimension $n^{2}$ over its centre $F$. The main result of [1], asserts that if $t=n^{2}$, then as $F$-algebras

$$
\lim _{i \rightarrow \infty} M_{t^{i}} D \simeq \lim _{i \rightarrow \infty} M_{t^{i}} F
$$

the maps in both limits being $\Delta(t)$. However, the proof there is exceptionally obscure and complicated. We give a short natural proof, requiring only the Noether-Skolem Theorem:

[3, TheOREM 4.3.1]. Let $C$ be a finite dimensional simple $F$-algebra with centre $F$, and let $A, B$ be simple subalgebras of $C$, each with centre $F$. Then any $F$-algebra isomorphism from $A$ to $B$ can be extended to an inner automorphism of $C$.

ThEOREM. Let $D$ be a division algebra of dimension $n^{2}$ over its centre, the field $F$. Set $t=n^{2}$, and for each positive integer $i$, let $j_{i}$ denote the map $M_{t} F \subset M_{t} D$ induced by the inclusion of $F$ in $D$. Form the $F$-algebras $\lim _{i \rightarrow \infty} M_{t} i F, \lim _{i \rightarrow \infty} M_{t} i D$, with $\Delta(t)$ as the maps in the limits.

There exist inner automorphisms $\psi_{i}, \varphi_{i}$ of $M_{t} D, M_{t} F$ respectively, so that if $\alpha_{i}: M_{t} F \rightarrow M_{i} D$ are defined by $\alpha_{i}=\psi_{i}^{-1} j_{i} \varphi_{i}$, then the $\alpha_{i}$ are compatible with the maps in the direct limits, and the induced map

$$
\lim _{i \rightarrow \infty} \alpha_{i}: \lim M_{t^{i}} F \rightarrow \lim M_{t^{i}} D
$$

is an F-algebra isomorphism.

Received by the editors May 31, 1977.

AMS (MOS) subject classifications (1970). Primary 16A40. 
Let $k: D \rightarrow M_{t} F$ be a fixed $F$-algebra homomorphism (for instance, the right regular representation of $D$ ), and define maps $k_{i}: M_{t} D \rightarrow M_{t^{i+1}} F$ to be the maps on the matrix rings induced by $k$. We may form the limit, $S$, of the diagram (1):

$$
F \stackrel{j_{0}}{\rightarrow} D \stackrel{k}{\rightarrow} M_{t} F \stackrel{j_{1}}{\rightarrow} M_{t} D \stackrel{k_{1}}{\rightarrow} M_{t^{2}} F \stackrel{j_{2}}{\rightarrow} \ldots
$$

Then $S$ is algebra isomorphic to $\lim _{i \rightarrow \infty} M_{t} F$.

Proof. Pick a fixed map $k$, such as the right regular representation, and separate (1) into two rows, rows 2 and 3 of diagram (2).

$$
\begin{aligned}
& \cdots \rightarrow M_{t^{i}} F \longrightarrow M_{t^{i+1}} F \longrightarrow M_{t^{i+2}} F \rightarrow \cdots \\
& \cdots \rightarrow M_{t^{i}} F \stackrel{k_{i} j_{i}}{\longrightarrow} M_{t^{i+1}} F \stackrel{k_{i+1} j_{i+1}}{\longrightarrow} M_{t^{i+2}} F \rightarrow \cdots \\
& \cdots \rightarrow M_{t^{i}} D \stackrel{j_{i+1} k_{i}}{\longrightarrow} M_{t^{i+1}} D \longrightarrow M_{t^{i+2}} D \rightarrow \cdots \\
& \cdots \rightarrow M_{t^{i}} D \longrightarrow M_{t^{i+2}} D \rightarrow \cdots
\end{aligned}
$$

Because $M_{t} F$ and $M_{t} D$ are cofinal in diagram (1), $\lim j_{i}$ is actually an isomorphism (with inverse, $\lim k_{i}$ ) from the limit of row 2 to the limit of row 3. We shall construct inner $\varphi_{i}: M_{t} F \rightarrow M_{t^{i}} F$ (row 1 to row 2) and $\psi_{i}: M_{t^{i}} D \rightarrow$ $M_{i} D$ (row 4 to row 3 ) so that the whole of (2) commutes.

Define $\psi_{0}: D \rightarrow D$ to be the identity map. Assuming $\psi_{s}$ have been defined for $0 \leqslant s \leqslant i$, so that rows 3 and 4 commute, we see

$$
j_{i+1} k_{i} \psi_{i}\left(M_{t^{i}} D\right) \simeq \Delta\left(M_{t^{i}} D\right)
$$

as $F$-subalgebras of $M_{t^{i+1}} D$, the isomorphism obtained by pulling back the image of $\Delta$, and applying $j_{i+1} k_{i} \psi_{i}$. By the Noether-Skolem Theorem, there exists an invertible $V$ in $M_{t^{i+1}} D$ so that this isomorphism is implemented by conjugation with $V$. Define $\psi_{i+1}(A)=V A V^{-1}$; then $\psi_{i+1} \Delta=j_{i+1} k_{i} \psi_{i}$, concluding the induction.

Thus $\lim \psi_{i}$ defines a map between the limits of the fourth and third rows; it follows that $\lim \psi_{i}^{-1}$ (from row 3 to row 4) exists and is the inverse. In particular, $\lim \psi_{i}^{-1}$ is an algebra isomorphism.

The same process allows us to construct a similar isomorphism, $\lim \varphi_{i}$ from the limit of row 1 to the limit of row 2 , with each $\varphi_{i}$ inner. Since $\lim j_{i}$ is an isomorphism, and

$$
\lim \left(\psi_{i}^{-1} j_{i} \varphi_{i}\right)=\left(\lim \psi_{i}^{-1}\right)\left(\lim j_{i}\right)\left(\lim \varphi_{i}\right),
$$

setting $\alpha_{i}=\psi_{i}^{-1} j_{i} \varphi_{i}$ (mapping down the columns of (2)), we see that $\lim \alpha_{i}$ is an isomorphism, and the final statement is an immediate consequence.

The form of the isomorphism obtained above is particularly useful in computing the homology or cohomology of $D$ relative to that of $F$ (see [2], for 
an application), because such functors usually commute with direct limits, and change inner automorphisms into the identity.

Theorem 2 of [1] effectively asserts that if a division ring $D$ can be represented as a $\operatorname{limit}, \lim D_{i}$, with each $D_{i}$ a finite dimensional central division algebra over $F$, then

$$
\lim _{\Delta(m)} M_{n} F \simeq \lim _{\Delta(m)} M_{n} D
$$

where $m$ varies over all products of numbers of the form $\left[D_{i}: F\right]$, and $n$ varies similarly. (Of course, $\Delta(m): M_{n} F \rightarrow M_{r} F$ is defined only if $r=m n$.) One can easily prove that this follows from our theorem above.

\section{REFERENCES}

1. B. P. Dawkins and I. Halperin, The isomorphism of certain continuous rings, Canad. J. Math. 18 (1966), 1333-1344; Corrigendum, ibid. 20 (1968), 512.

2. S. Green, D. Handelman and P. Roberts, K-theory of finite dimensional algebras, J. Pure Appl. Algebra (to appear).

3. I. Herstein, Noncommutative rings, Carus Math. Monographs, no. 15, Wiley, New York, 1968.

Department of Mathematics, University of Utah, Salt Lake Ctry, Utah 84112

Current address: Department of Mathematics, University of Ottawa, Ottawa, Ontario, Canada K1N 6N5 